\title{
Feasibility of an FDM System Using Mach-Zehnder Filters in a Smart Grid
}

\author{
Yuki Fukushima*, Isamu Wakabayashi*, Xiaoxuan Che**, Masatoshi Sano* \\ *Tokyo University of Science, Electrical Engineering Department \\ **Wuhan University, Institute of Advanced Energy \\ wakaba@ee.kagu.tus.ac.jp
}

\begin{abstract}
The present paper describes the feasibility of the FDM system using Mach-Zehnder filters for a power control system in a smart grid. The interconnection of large quantities of the distributed power sources, such as PV power sources, to the current bulk power grids is anticipated, and as a result, fluctuations of voltage and frequency will increase and make the adjustment of the power demand-supply balance difficult. By applying information communication technology to a power supply system in order to control the digital grid routers, energy-savings, and a stable power supply, the widespread use of renewable energy is expected. The digital grid cell includes numerous consumers, such as houses, office buildings and factories, which may or may not have PV power sources. The proposed FDM system enables the processing of a great deal of information to be exchanged incoherently between consumers and power generators, because the FDM system has the capability of $2^{\mathrm{N}}$ transmission channels and the transfer functions of which can be expressed as the basis of the plain representation of the transfer function of the Mach-Zehnder filter. Using the FDM system, it will be possible to control a power supply system in a smart grid in order to maintain a stable power supply, energy savings, and a suitable adjustment of the power demand-supply balance.
\end{abstract}

Keywords-FDM, Mach-Zehnder filter, Smart grid, Digital grid cell, PV power generator

\section{INTRODUCTION}

The present paper describes the feasibility of an FDM system using Mach-Zehnder filters (MZFs) for a power control system in a smart grid where a digital grid cell system is introduced.

The Kyoto Protocol of the United Nations Framework Convention on Climate Change came into effect in order to prevent global warming due to greenhouse gases, such as carbon dioxide, in December-1997. Since then, a great deal of research on energy-savings has been conducted in various fields.

Since the Fukushima I nuclear power plant was damaged by the tsunami generated by the Great East Japan
Earthquake in March-2011, power shortages have occurred, and the introduction of photovoltaic (PV) power generation as a renewable energy has come to be expected furthermore.

However, the interconnection of large quantities of distributed power sources, such as PV power sources, to the current bulk power grids causes an increase in voltage and frequency fluctuations which make the adjustment of the power demand-supply balance difficult, because the PV and the wind power generators of distributed power sources depend on changes in climate conditions. In order to promote the introduction of renewable energy, a new power supply system is required. We consider a smart grid to be integral to the realization of such a system. A digital grid [1] and informatization of energy [2,3] have also been proposed as components of such a system. Common specifications of smarttaps [4], on-demand power supply [5], power coloring [6], power packetization, and routing technology [7-9] have been examined practically. The widespread use of renewable energy is expected by applying information communication technology (ICT), such as time division multiplexing and wavelength division multiplexing schemes in digital transmission systems, to a power supply system in order to control the digital grid routers, energy-savings, and a stable power supply [10].

We have presented frequency division multiplexing (FDM) system architectures using Mach-Zehnder filters (MZFs) based on the analysis techniques of periodic branching filters (PBFs) [11]. The PBF was developed as a microwave multi/demultiplexer and has been used in communication satellites [12]. Another type of filter, the MZF, which is described in Appendix A, is used as an optical filter and an intensity modulator in optical communication applications [13-15]. Although the PBF and the MZF have been developed separately in the respective fields, the basic structure and the operating principle of the MZF can be thought of as being similar to those of the PBF $[16,17]$.

An FDM system has contributed to increased transmission capacity and is used in optical communication system as a wavelength division multiplexing system [18-20]. However, thus far, analyzing the transmission characteristics for the case in which the system has a much higher number of channels is difficult. 
In section II, a power supply system using digital grid cells is shown to solve a current subject. In section III, we present an FDM system configuration in the case of a four-channel system as an example of a $2^{\mathrm{N}}$-channel FDM system $[21,22]$ and describe the amplitude transfer function of the system. The signal waveform and the inter-channel interference ratio are also shown in order to demonstrate the system performance. In section IV, as an example, the smart grid system applying the FDM system is presented schematically. Finally in section V, a summary is presented.

\section{POWER SuPPly SySTEM}

\section{A. Current Bulk Power Supply System}

According to the current bulk power grids, the electric power is supplied from the power plants to consumers through various voltage substations and the pole transformer. Some of the consumers, such as houses, office buildings, stores, and small factories are supplied with the renewable energy such as photovoltaic generated power. The power supply equipment is controlled in order to maintain a stable power supply by the centralized control center.

The interconnection of large quantities of PV power sources to the current bulk power grids is anticipated. That causes an increase in voltage and frequency fluctuations. The current system under the centralized management control system will be unable to cope with difficulties, such as those fluctuations and a massive power failure.

\section{B. Power Supply System Using Grid Cells}

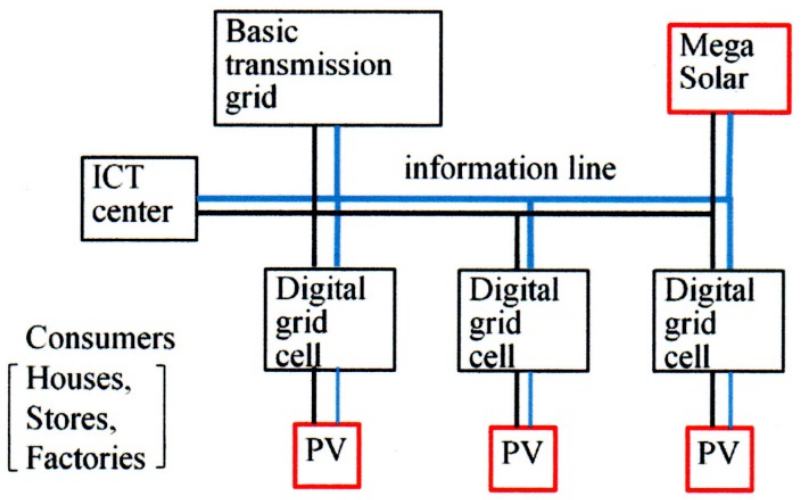

Figure 1. A power supply system using digital grid cells.

Figure 1 illustrates schematically the power supply system using a digital grid cell that is considered in order to penetrate a PV generated power. A basic transmission grid represents a current bulk power supply system from the power plant to mainly the pole transformer. A conventional wide-area synchronized grid is subdivided into small or medium sized cells, and connected through asynchronous and coordinated control power exchanges and the subdivided grid is called digital grid cell as in [1]. Some of the consumers inside the cell have PV generators. The cell itself is possible to have a PV generator. A power line and an information line are, respectively, indicated by a black line and a blue line. We have considered whether our proposed FDM system [21,22] is applicable to this information line system.

\section{III. $2^{\text {N}}$-CHANNEL FDM SySTEM}

\section{A. System Configuration}

As an example of a $2^{\mathrm{N}}$-channel FDM system [23] for simplicity, the configuration of the four-channel FDM system is illustrated in Figure 2 [22]. This system consists of three MZFs (MZF is described in Appendices A, B, C.) on the transmit side and three MZFs on the receive side and the MZFs are reciprocal. The notched symbol represents the matched termination.

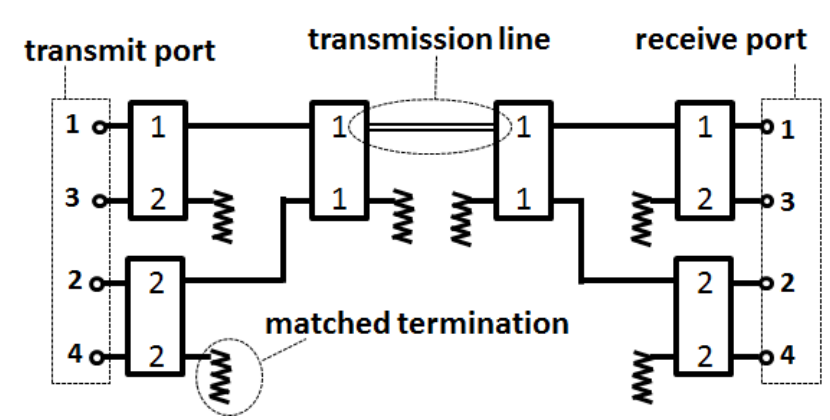

Figure 2. A four-channel FDM system configuration.

The transfer functions between the transmit port and the receive port in Figure 1 can be expressed using equations in Appendices $\mathrm{C}$ and $\mathrm{B}$ as follows:

$$
\begin{aligned}
& H_{1 \rightarrow r}(\omega)=C(1,2) C(1,1) C(r, 1) C(r, 2), \\
& H_{2 \rightarrow r}(\omega)=C(2,2) C(2,1) C(r, 1) C(r, 2), \\
& H_{3 \rightarrow r}(\omega)=C(3,2) C(3,1) C(r, 1) C(r, 2), \\
& H_{4 \rightarrow r}(\omega)=C(4,2) C(4,1) C(r, 1) C(r, 2) .
\end{aligned}
$$

A subscript $1 \rightarrow \mathrm{r}$ in the left hand side of equation (1) indicates that an input signal transmits from transmit port 1 to receive port $r$, where $r=1,2,3,4$.

In the case of $2^{\mathrm{N}}$-channel FDM system in general, the transfer function from the transmit port $\mathrm{n}$ to the receive port $\mathrm{r}$ can be represented in equation (5),

$$
\begin{aligned}
H_{n \rightarrow r}(\omega) & =C\left(n, 2^{N-1}\right) C\left(n, 2^{N-2}\right) C\left(n, 2^{N-3}\right) \cdots C(n, 4) C(n, 2) C(n, 1) \\
& \times C(r, 1) C(r, 2) C(r, 4) \cdots C\left(r, 2^{N-3}\right) C\left(r, 2^{N-2}\right) C\left(r, 2^{N-1}\right) \\
& =\prod_{k=0}^{N-1} C\left(n, 2^{k}\right) C\left(r, 2^{k}\right)
\end{aligned}
$$

where the fixed carrier phase and delay time are omitted here. The transmission line is assumed to be lossless and non-dispersive. Channels between identical port numbers are assumed to be desired channels, and channels between different port numbers are assumed to be interference channels. 


\section{B. Inter-Channel Interference Ratio}

Here, the channel 1 is assumed to be the desired channel. The transmission scheme is assumed to be ASK. The transmit signal, $\mathrm{g}_{\mathrm{T} 1}(\mathrm{t})$, at port 1 is represented by equation (6). The signals from transmit port $\mathrm{n}$ to receive port 1 are considered to interfere with the signal from transmit port 1 . Owing to the incoherent transmission, the transmit signal, $\mathrm{g}_{\mathrm{Tn}}(\mathrm{t})$, at port $\mathrm{n}$ is random process and is given by equation (7).

$$
\begin{aligned}
& g_{T 1}(t)=\sum_{k=-\infty}^{\infty} a_{k} g(t-k T) \cos \omega_{1} t \\
& g_{T n}(t)=\sum_{k=-\infty}^{\infty} b_{n k} g\left(t-k T+\xi_{n}\right) \cos \left(\omega_{n} t+\phi_{n}\right)
\end{aligned}
$$

where $a_{k}$ and $b_{n k}$ is the independent and identically distributed (i.i.d.) random variables that take 1 or $0 . \xi_{n}$ and $\phi_{\mathrm{n}}$ are uniformly distributed over the range of $[0, \mathrm{~T}]$ and $[0,2 \pi]$, respectively. $g(t)$ is a rectangular pulse with unit amplitude and a pulse width of $\mathrm{T}$, which is the symbol period. The frequency spectrum of a single pulse, $g(t)$, is given as follows:

$$
G(\omega)=T \operatorname{sinc}\left(\frac{\omega}{\omega_{r}}\right)
$$

where $\omega_{\mathrm{r}}(=2 \pi / \mathrm{T})$ is the clock radian frequency. The frequency spectrum $G_{R 1}(\omega)$ of the received pulse at desired channel 1 is given as follows:

$$
G_{R 1}(\omega)=G(\omega) \cdot H_{1 \rightarrow 1}(\omega)
$$

Thus, the received pulse $\mathrm{g}_{\mathrm{R} 1}(t)$ can be obtained by the inverse Fourier transform of $G_{R 1}(\omega)$ given as equation (10). The baseband signal, $g_{R}(t)$, can be expressed by equation (11).

$$
\begin{aligned}
& g_{R 1}(t)=\sum_{k=-\infty}^{\infty} a_{k} g_{R}(t-k T) \cos \omega_{1} t \\
& g_{R}(t)=\frac{1}{2^{2 N}} \sum_{k=-\left(2^{N}-1\right)}^{2^{N}-1}\left(2^{N}-|k|\right) g\left(t-\frac{2 k}{2^{N}} T_{d}\right)
\end{aligned}
$$

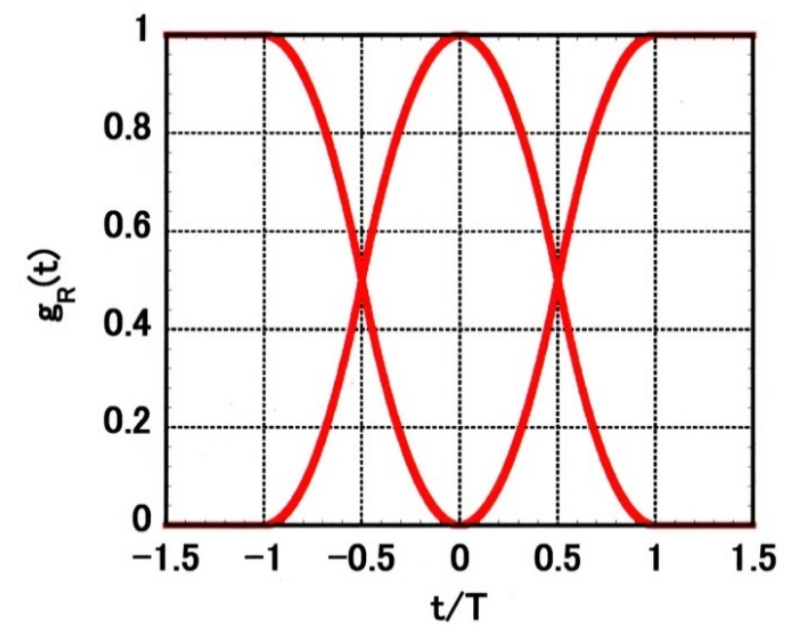

Figure 3. Eye pattern of desired channel $1(\mathrm{~N}=10)$.

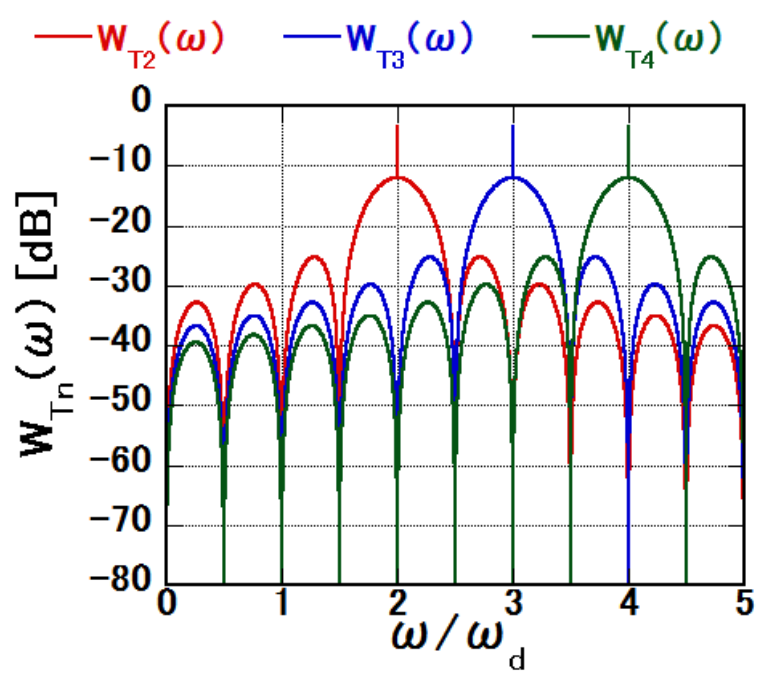

Figure 4. Transmit signal PSDs.

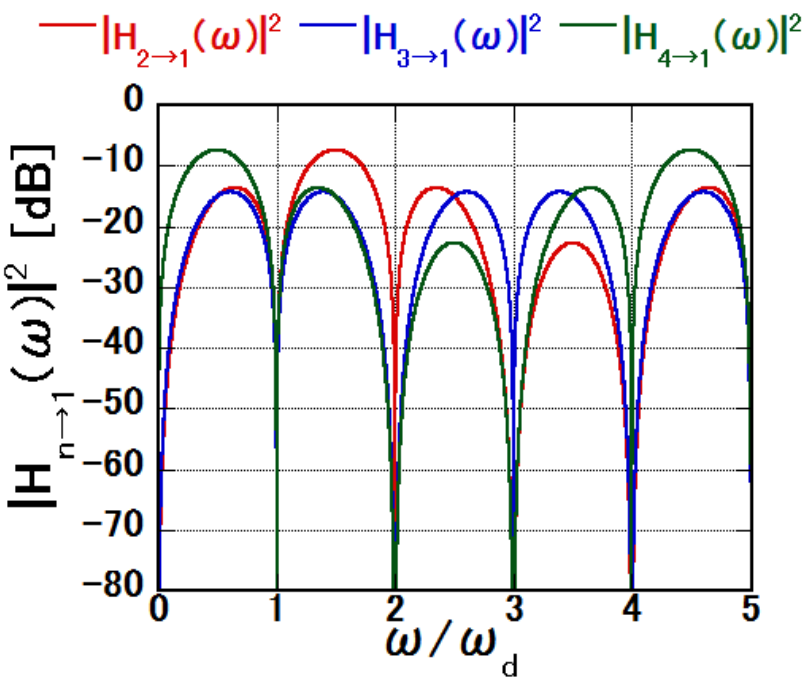

Figure 5. Transmission characteristics at the interference paths.

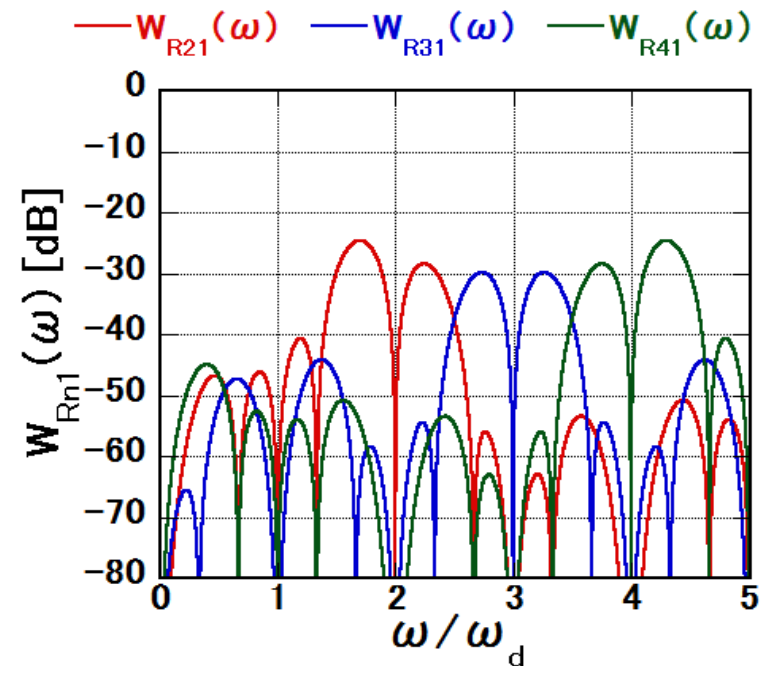

Figure 6. The interference signal PSDs at the receive port 1. 
Figure 3 shows the eye pattern of deterministic signal at desired channel 1 when $2^{10}$-channel system is used with $\omega_{\mathrm{d}} / \omega_{\mathrm{r}}\left(=\mathrm{T} / 2 \mathrm{~T}_{\mathrm{d}}\right)$ of 2.0. The carrier wave is omitted here. The power spectral density (PSD) of $\mathrm{g}_{\mathrm{Tn}}(\mathrm{t})$ can be denoted as $\mathrm{W}_{\mathrm{Tn}}(\omega)$, and given as follows:

$W_{T n}(\omega)=\frac{1}{16 T}\left\{\left|G\left(\omega-\omega_{n}\right)\right|^{2}+\left|G\left(\omega+\omega_{n}\right)\right|^{2}\right\}+\frac{\pi}{8}\left\{\delta\left(\omega-\omega_{n}\right)+\delta\left(\omega+\omega_{n}\right)\right\}$

where $G(\omega)$ is the frequency spectrum of $g(t)$, and $\delta(\omega)$ is the Dirac delta function. The interference signal PSDs at receive port $\mathrm{r}$ from transmit port $\mathrm{n}$ can be denoted as $\mathrm{W}_{\mathrm{Rnr}}(\omega)$. The PSD is given as follows:

$$
W_{R n r}(\omega)=\left|H_{n \rightarrow r}(\omega)\right|^{2} W_{T n}(\omega)
$$

Figure 4 shows the signal PSDs as given in equation (12) at transmit ports $n=2,3$, and 4 as a function of $\omega / \omega_{d}$, where $\omega_{\mathrm{d}}$ is a neighboring channel spacing. Figure 5 shows the transmission characteristics $\left|\mathrm{H}_{2 \rightarrow 1}(\omega)\right|^{2}$, $\left|\mathrm{H}_{3 \rightarrow 1}(\omega)\right|^{2}$, and $\left|\mathrm{H}_{4 \rightarrow 1}(\omega)\right|^{2}$ given as in square of equation (5) along the interference paths as a function of $\omega / \omega_{\mathrm{d}}$. Figure 6 shows the interference signal PSDs at receive port $\mathrm{r}=1$ from transmit ports $\mathrm{n}=2,3$, and 4 as a function of $\omega / \omega_{\mathrm{d}}$. These PSDs can be obtained as the product of the transmit signal PSDs and the transmission characteristics, as given in equation (13).

The interference power $P_{R n r}$ at receive port $\mathrm{r}$ can be obtained as follows:

$$
P_{R n r}=(1 / 2 \pi) \int_{-\infty}^{\infty} W_{R n r}(\omega) d \omega
$$

The signal to inter-channel interference ratio (ICI) at receive port $\mathrm{r}$ can be expressed as follows:

$$
\rho_{r}=A_{e}^{2} /\left(2 \sum_{n=2}^{2^{N}} P_{R n r}\right)
$$

where $A_{e}$ is an eye aperture. Figure 7 shows the calculated results of ICI ratio at receive port $r=1$ in the case of 2-channel, 4-channel, 8-channel, and $2^{10}$-channel FDM system.

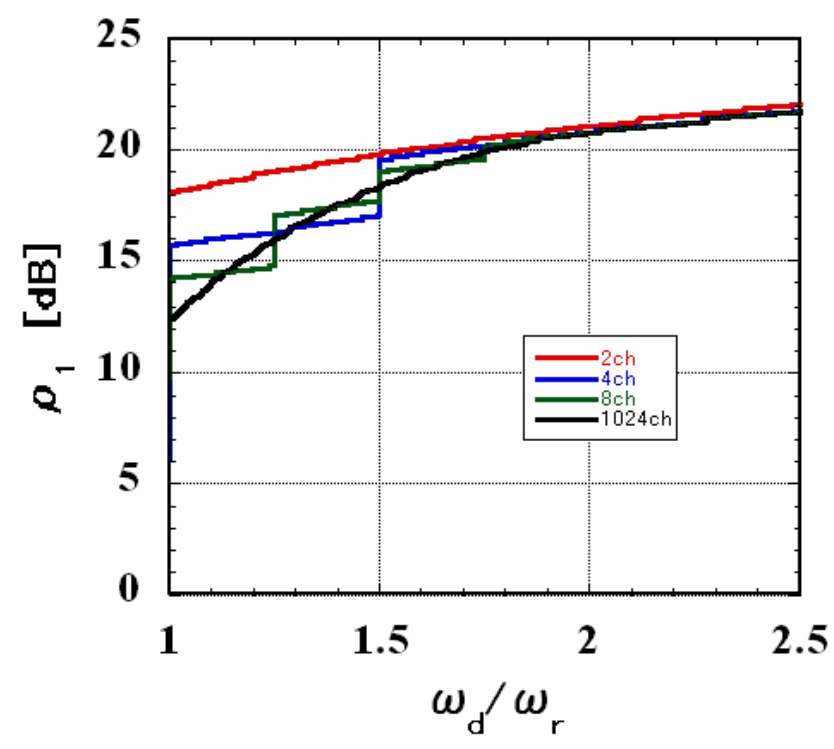

Figure 7. A signal to inter-channel interference ratio at the receive port 1.

\section{APPLICATION}

Figure 8 illustrates a schematic diagram of a smart grid. The FDM system for the information line is represented by the bidirectional thick arrows between routers. The constitutive elements are a basic transmission line router that is connected to a basic transmission grid, digital grid cell routers, and home routers including central information center. Each router has functions of controlling and maintaining power savings, and a stable power supply. Each cell includes houses, office buildings, stores, and small factories, which may or may not have PV solar cells and is working independently. The storage batteries and power conditioners are usually arranged adjacent to the renewable energy sources of PV generators. Inside home, we have electric appliances such as TV, PC, refrigerator and so on. These appliances with smart taps may be connected to the home router through the FDM system. In the same way, each home may be connected to the cell router through the FDM system. Each cell may be connected with each other through the router. The routers in a smart grid treat numerous data to be exchanged incoherently.

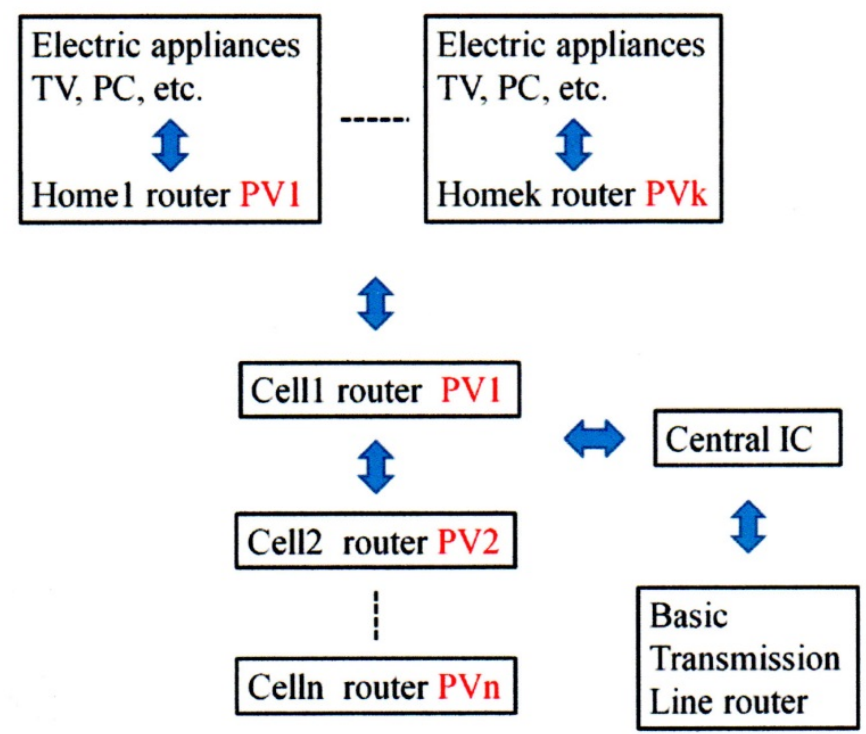

Figure 8. A schematic diagram of a smart grid.

The general concept of the smart grid is to unify the information of both consumers and distributed-type renewable energy sources to realize a highly efficient, high-quality, highly reliable power supply system together with the current concentrated-type power supply system, such as thermal power generators, by making full use of information communication technology [24]. Based on this concept, the FDM system presented here is available for maintaining a stable power supply, energy savings, and a suitable adjustment of the power demand-supply balance because the proposed FDM system has the capability of $2^{\mathrm{N}}$ transmission channels, and as a result, it enables the processing of a great deal of information to be exchanged 
incoherently between consumers and power generators, and because the transfer functions of which can be expressed as the basis of the plain representation of the transfer function of the Mach-Zehnder filter.

\section{SUMMARY}

The present paper described the applicability of the FDM system using Mach-Zehnder filters for a power control system in a smart grid where a digital grid cell system would be introduced.

The widespread use of renewable energy is expected by applying ICT technology to a power supply system in order to control and maintain the home energy-savings, a stable power supply and a suitable adjustment of a power demand-supply balance. In this situation, the proposed FDM system will be available for processing a great deal of information to be exchanged incoherently among power generators, cells, and consumers, because the FDM system has the capability of $2^{\mathrm{N}}$ transmission channels, and the transfer functions of which can be expressed as the basis of the plain representation of the transfer function of the Mach-Zehnder filter.

In the future, we intend to consider the band-limitation method of the proposed FDM system, such as cosine roll-off characteristics and then to design the actual FDM system and to perform its simulation in order to clarify the power supply system performance quantitatively.

\section{ACKNOWLEDGMENT}

The authors would like to thank Prof. Kazuhiro Miyauchi for his valuable guidance and support.

\section{REFERENCES}

[1] R. Abe, H. Taoka, D. McQuilkin, "Digital Grid: Communicative Electrical Grids of the Future," IEEE Trans. on Smart Grid, Vol. 2, No. 2, pp. 399-410, June 2011.

[2] T. Matsuyama, "i-Energy: Background, Objective, Ideas, and Realization Methods," Information Processing Society of Japan (IPSJ) Magazine, Vol. 51, No. 8, pp. 926-933, August 2010.

[3] T. Kato, "Social Engineering Approach to Realize Informatization of Energy," IPSJ Magazine, Vol.51, No.8, pp.972-977, August 2010.

[4] M. Tsukamoto, T. Kato, "Developing Common Specifications of SmartTaps,” IPSJ Magazine, Vol. 51, No. 8, pp. 934-942, August 2010.

[5] Y. Okabe, "Quality of Energy Aware Routine toward Energy on Demand Home Electric Network," IPSJ Magazine, Vol. 51, No. 8, pp. 951-958, August 2010.

[6] Y. Okabe, "Research Topics on Smart Energy Management in Home Network," Proc. IEICE (Japan) Society Conference 2012, BP-4-3, SS-19, September 2012.

[7] T. Hikihara, "Power Packetization and Routing Technology," IPSJ Magazine, Vol. 51, No. 8, pp. 943-950, August 2010.

[8] R. Takahashi, K. Tashiro, T. Hikihara, "Feasibility Estimation of Power Packetization and Power Supply Based on Power Packet Dispatching System," Proc. IEICE General Conference 2013, BS-6-1, S-152, March 2013.

[9] Y. Miura, M. Eguchi, Y. Okui, Y. Nishida, T. Takeshita, T. Funabashi, "Power Electronics as Element Technology of New Power and Energy System with a Focus on Photovoltaic Generation," Proc.
IEE Japan General Conference 2013, 6-S8-5, S8(pp. 14-17), March 2013.

[10] I. Wakabayashi, X. Chen, M. Sano, "Feasibility of an FDM system Using Mach-Zehnder Filters for a Power Control System," Proc. of $9^{\text {th }}$ China SoG and Si PV Conference, Nov. 2013.

[11] K. Miyauchi, I. Ohtomo, S. Shimada, "A Transmission Type Periodic Branching Filter," Proc. four electrical engineers society's joint conference (Japanese ed.), No.1493, pp.1769-1770, 1969.

[12] H. Kumazawa, I. Ohtomo, "30-GHz-Band Periodic Branching Filter Using a Traveling-Wave Resonator for Satellite Applications," IEEE Trans. Microwave Theory Techniques, Vol.MTT-25, No.8, pp.683-687, August 1977.

[13] G. P. Agrawal, Fiber-Optic Communication System, third ed., K. Chang, Ed. New York, A John Wiley \& Sons, Inc., pp.123, and 342, 2002 .

[14] N. Takato, T. Kominato, A. Sugita, K. Jinguji, H. Toba, M. Kawachi, "Silica-Based Integrated Optic Mach-Zehnder Multi/Demultiplexer Family with Channel Spacing of 0.01-250nm," IEEE Journal of Selected Areas in Commun., Vol.8, No.6, August 1990

[15] T. Hanada, T. Shimoda, M. Kitamura, S. Nakamura, "FDM/WDM Couplers Using Silica Waveguide Deposited by APCVD," IEICE Trans. Electron., Vol.E80-C, No.1, pp.130-133, January 1997.

[16] K. Oda, N. Takato, H. Toba, K. Nosu, “A Wide-Band Guided-Wave Periodic Multi/Demultiplexer with a Ring Resonator for Optical FDM Transmission Systems," IEEE Journal of Lightwave Technology, Vol.6, No.6. pp.1016-1023, June 1988.

[17] K. Nosu, H. Toba and K. Iwashita, "Optical FDM Transmission Technique,” IEEE Journal of Lightwave Technology, Vol.LT-5, No.9, pp.1301-1308, September 1987.

[18] K. Inoue, N. Takato, H. Toba, M. Kawachi, "A Four-Channel Optical Waveguide Multi/Demultiplexer for 5-GHz Spaced Optical FDM Transmission," IEEE Journal of Lightwave Technology, Vol.6, No.2, pp.339-345, February 1988.

[19] S.-H. Lee, Y.-Y. Won, H.-D. Jung, S.-K. Han, "Reduction of Inter-Channel Crosstalk Using Mach-Zehnder Type Filter in Digital/RF Optical Transmission Link," IEE Proc. Optoelectron., Vol.152, No.4, pp.189-192, August 2005.

[20] B. Charbonnier, et al., "Silicon Photonics for Next Generation FDM/FDMA PON," J. OPT. COMMUN. NETW., Vol.4, No.9, pp.A29-A37. September 2012.

[21] T. Sekiguchi, I. Wakabayashi, S. Seki, K. Miyauchi, "Transmission Characteristics of 2-Channel FDM System Using Mach-Zehnder Filters," Proc. IEICE Society Conference 2004, B-10-75, September 2004.

[22] K. Tanaka, I. Wakabayashi, M. Sano, "Transmission Characteristics of an FDM System Using Mach-Zehnder Filters," $15^{\text {th }}$ Proceedings of ICACT 2013, Vol.1, pp.89-93, January 2013.

[23] Y. Fukushima, I. Wakabayashi, M. Sano, "Transmission Characteristics of $2^{\mathrm{N}}$-Channel FDM System Using Mach-Zehnder Filters," Proc. IEICE Society Conference 2013, B-10-84, September 2013.

[24] G. Ushio, "Smart-Grid Concept and Issues Surrounding Electric Power System," Proc. IEE Japan General Conference 2013, 6-S11-2, S11(pp.3-6), March 2013.

\section{APPENDICES}

\section{Appendix A. Mach-Zehnder Filter Basic Configuration}

Figure A1 shows the Mach-Zehnder Filter (MZF) basic configuration. The MZF consists of two 3-dB couplers with two input/output ports and two optical transmission lines between the two couplers. The lengths of optical transmission lines A and $B$ are assumed to be $L_{1}$ and $L_{2}$, respectively. Both of these lines are assumed to have a phase constant of $\beta_{0}$ and a delay time per unit length of $\tau_{0}$ at the center radian frequency.The filter is assumed to be reciprocal and lossless with no dispersion herein. The fixed 
phase change and delay time are ignored. The coupling coefficient $\mathrm{k}$ of the two $3-\mathrm{dB}$ couplers is $1 / \sqrt{2}$.

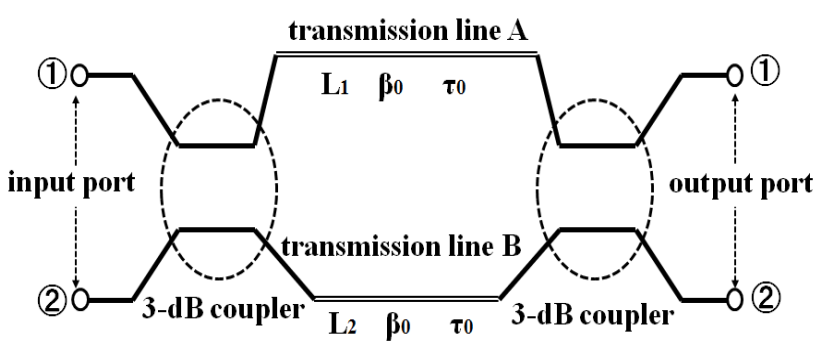

Figure A1. MZF basic configuration.

The first coupler splits the input signal equally into two parts, which acquire different phase shifts, when the transmission line (arm) lengths are made different, before they interfere at the second coupler [14]. Since the relative phase shift is frequency dependent, the transmittivity is also frequency dependent. The two arm signals are recombined at the second $3-\mathrm{dB}$ coupler. Thus, the transmission characteristics of the MZF, as well as those of the peoriodic branching filter $(\mathrm{PBF})$, change periodically depending on frequency.

\section{Appendix B. Transfer Functions of MZF}

The transfer functions $A_{i \rightarrow 0}(\omega)$ and $\theta_{i \rightarrow 0}(\omega)$ of the MZF from input port $i$ to output port o can be expressed as follows:

$$
\begin{aligned}
\mathrm{A}_{1 \rightarrow 1}(\omega) & =\cos \frac{\pi\left(\omega-\omega_{1}\right)}{2 \omega_{d}}, \\
\mathrm{~A}_{1 \rightarrow 2}(\omega) & =\cos \frac{\pi\left(\omega-\omega_{2}\right)}{2 \omega_{d}}, \\
\mathrm{~A}_{2 \rightarrow 1}(\omega) & =\cos \frac{\pi\left(\omega-\omega_{2}\right)}{2 \omega_{d}}, \\
\mathrm{~A}_{2 \rightarrow 2}(\omega) & =\cos \frac{\pi\left(\omega-\omega_{1}\right)}{2 \omega_{d}}, \\
\theta_{1 \rightarrow 1}(\omega) & =\theta_{1 \rightarrow 2}(\omega)=\theta_{2 \rightarrow 1}(\omega)=\theta_{2 \rightarrow 2}(\omega) \\
& =-\frac{\beta(\omega) \mathrm{L}}{2}+\frac{\pi}{2} .
\end{aligned}
$$

The phase constant $\beta(\omega)$ is approximated to the first degree, and $\Delta \mathrm{L}$ and $\mathrm{L}$ are defined as follows:

$$
\begin{aligned}
& \beta(\omega)=\beta_{0}+\tau_{0}\left(\omega-\omega_{\mathrm{n}}\right) \quad ; n=1,2, \\
& \Delta \mathrm{L}=\mathrm{L}_{1}-\mathrm{L}_{2}, \\
& \mathrm{~L}=\mathrm{L}_{1}+\mathrm{L}_{2}, \\
& \tau_{0} \Delta \mathrm{L}=\frac{\pi}{\omega_{\mathrm{d}}}=\mathrm{T}_{\mathrm{d}},
\end{aligned}
$$

where $\omega_{1}$ and $\omega_{2}$ are the center radian frequency of input ports 1 and 2, respectively, in Figure A1. $T_{d}$ is the difference in delay time between the two arms, and $\omega_{\mathrm{d}}$ is the neighboring channel spacing. Finally, $\beta_{0} \Delta \mathrm{L}$ is an odd multiple of $\pi$ with respect to $\omega_{1}$ and an even multiple of $\pi$ with respect to $\omega_{2}$.

\section{Appendix C. MZF Model}
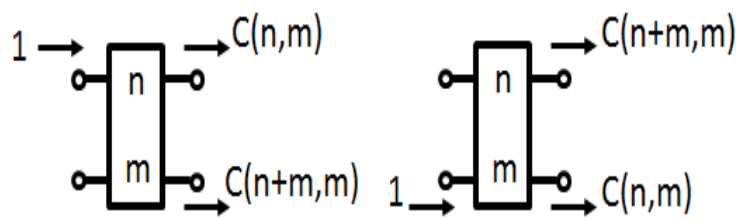

Figure C1. MZF model.

Figure $\mathrm{C} 1$ shows the MZF model. Based on equations (B1) through (B4), a plain representation of the amplitude transfer function of the MZF can be further defined as follows:

$$
\begin{aligned}
& C(n, m)=\cos \frac{\pi\left(\omega-\omega_{n}\right)}{2 m \omega_{d}}, \\
& \omega_{n}=\omega_{1}+(n-1) \omega_{d}, \\
& C(n, m)=C(n+2 v m, m)
\end{aligned}
$$

where $\omega_{\mathrm{n}}$ is the center radian frequency at channel $\mathrm{n}, \mathrm{m} \omega_{\mathrm{d}}$ is the channel spacing, and $v$ is an integer. 